Recherches en didactique des langues et des cultures

Les cahiers de l'Acedle

17-2 | 2020

Recherches collaboratives en didactique des langues

\title{
De l'utilité de la recherche collaborative pour produire des ressources de formation robustes
}

\section{Bernadette Kervyn}

\section{OpenEdition}

Journals

Édition électronique

URL : https://journals.openedition.org/rdlc/7339

DOI : $10.4000 /$ rdlc.7339

ISSN : 1958-5772

Éditeur

ACEDLE

Référence électronique

Bernadette Kervyn, «De l'utilité de la recherche collaborative pour produire des ressources de formation robustes ", Recherches en didactique des langues et des cultures [En ligne], 17-2 | 2020, mis en ligne le 27 avril 2020, consulté le 17 octobre 2022. URL : http://journals.openedition.org/rdlc/7339 ; DOI : https://doi.org/10.4000/rdlc.7339

Ce document a été généré automatiquement le 17 octobre 2022.

\section{c) (ㄱ)}

Creative Commons - Attribution - Pas d'Utilisation Commerciale - Pas de Modification 4.0 International - CC BY-NC-ND 4.0

https://creativecommons.org/licenses/by-nc-nd/4.0/ 


\title{
De l'utilité de la recherche collaborative pour produire des ressources de formation robustes
}

\author{
Bernadette Kervyn
}

1 Dans le présent article, $\mathrm{j}^{1}$ propose d'analyser en quoi l'inscription dans un processus de recherche collaborative contribue à produire des ressources robustes pour la formation des enseignants en didactique de l'écriture. Pour ce faire, je commencerai par présenter brièvement le projet d'ingénierie didactique dont la mise en œuvre, par le biais d'une recherche collaborative, sera étudiée. Je décrypterai l'objectif précis de ce projet en mettant en exergue le principe de robustesse, défini au travers d'une double exigence de validité scientifique et de validité et pertinence de terrain. Mettant ensuite en dialogue le processus de recherche engagé et des travaux existants sur la recherche collaborative et la recherche-action, et plus globalement sur les méthodes de recherche en éducation, j'étudierai les caractéristiques par lesquelles la recherche collaborative mise en œuvre permet de construire la robustesse recherchée. Ce faisant, plutôt qu'une redéfinition générale de la recherche collaborative, je chercherai à mettre au jour les traits de ce type de recherche susceptibles de constituer des conditions facilitatrices, voire déterminantes, pour produire des ressources robustes en didactique du français.

\section{Des résultats de la recherche LireÉcrireCP à la production de ressources pour la formation}

2 Le projet d'ingénierie didactique sur lequel je prends appui pour analyser le rôle ou l'apport de la recherche de type collaboratif a été initié suite à la recherche LireÉcrire $\mathrm{P}^{2}$ à laquelle j'ai pris part aux côtés de plus de 80 chercheurs. Cette recherche pluridisciplinaire, coordonnée par R. Goigoux, avait pour ambition de produire des connaissances scientifiques sur les caractéristiques des pratiques efficaces d'enseignement de la lecture et de l'écriture au cours préparatoire (CP). Il s'agissait pour ce faire d'identifier les tâches, les contenus et les formes d'enseignement ainsi que 
leurs progressions, leurs dosages et leurs combinaisons les plus à même de faire progresser les différents publics d'élèves (Goigoux, 2016 ; Revue française de pédagogie, 2016).

La communication à un public de formateurs des premiers résultats de cette recherche de grande ampleur a d'emblée fait émerger la difficulté à passer d'une logique de recherche à une logique de formation. Comment rendre les résultats compréhensibles et pertinents en contexte de formation professionnelle ? Parmi les résultats, quels sont ceux qui demandent à priori un travail de transposition didactique voire de production de ressources spécifiques pour être ajustés et influents auprès des formateurs et des enseignants? Quels éléments issus de la recherche représentent un potentiel de développement professionnel important et à quelles conditions ?

Plutôt que de confier ou de déléguer toute la tâche de transposition et d'opérationnalisation (Kervyn, 20083) des résultats aux formateurs et aux enseignants, a été actée au sein du groupe de travail LireÉcrireCP la poursuite des investigations pour adosser à cette recherche empirique un axe formation via la production de ressources pour les formateurs et les enseignants de CP. Trois équipes de travail se sont ainsi constituées, dont une que j'ai coordonnée, centrée sur le volet écriture ${ }^{4}$. Réalisée de mai 2017 à février 2020, la recherche collaborative engagée sur ce volet écriture a été menée avec une équipe de formateurs exerçant en Aquitaine ainsi qu'avec le Centre Alain Savary (CAS) de l'Institut français de l'Éducation (IFÉ). Ce projet a consisté en la production de 4 scénarios de formation sur l'enseignement de l'écriture au CP ainsi que de ressources d'interface plus générales sur le processus scriptural et son enseignement, et sur les résultats de la recherche LireÉcrireCP en matière d'écriture (annexe 1) ${ }^{5}$. Au terme de ce long processus d'ingénierie didactique qui a permis de mettre à disposition sur le site du CAS ces différentes ressources complémentaires ${ }^{6}$, je souhaite revenir sur cette recherche de type collaboratif pour en analyser les objectifs et les caractéristiques.

\section{Une double exigence conjointe comme pilier de la robustesse des ressources}

5 Chercher à ce que les formateurs et les enseignants tiennent compte des résultats de la recherche LireÉcrireCP dans leurs pratiques en nous engageant dans la production de ressources m'a amenée à me questionner sur les critères de qualité essentiels pour les outils visés. Outre ceux assez diffusés d'utilité, d'utilisabilité et d'acceptabilité issus de l'ergonomie (Tricot et al., 2003), sur lesquelles je vais revenir, il me semble que la qualité dépend notamment de l'exigence scientifique. Autrement dit, il s'agit de considérer que le processus d'opérationnalisation pour le double terrain de la formation et de la classe ne doit pas se faire au détriment de la validité scientifique.

En termes de résultats, il est ainsi nécessaire qu'aux yeux des chercheurs, les ressources produites, non seulement ne soient pas en contradiction avec des connaissances scientifiques majeures ou consensuelles, mais qu'elles prennent en compte voire développent les connaissances actuelles sur l'écriture, sur son enseignement et son apprentissage en contexte scolaire ainsi que sur l'ingénierie didactique. En ce qui concerne le processus de recherche collaborative engagée, cette exigence nécessite notamment d'intégrer, outre les résultats de la recherche LireÉcrireCP, des objets et des 
résultats plus larges, issus de la didactique ainsi que de disciplines contributoires (principalement les sciences du langage, la psychologie, les sciences de l'éducation et l'ergonomie). L'exigence de scientificité nécessite aussi de réaliser une veille scientifique, de développer une culture scientifique commune aux chercheurs et aux praticiens, de mettre en œuvre une méthodologie de recherche en cohérence avec l'objectif, de mener un travail rigoureux à chaque étape de conception.

7 Néanmoins, cette exigence scientifique, aussi importante soit-elle, n'est pas en mesure de garantir à elle seule la qualité des outils : destinés aux professionnels formateurs et enseignants, ils se doivent d'intégrer les caractéristiques des utilisateurs, les exigences et les contraintes de leur métier et de leur milieu. Comme le rappelle Goigoux (2017), faute de prise en compte suffisante du public cible, ou faute d'ajustement à ce public, nombre d'outils ne se sont pas diffusés ou n'ont pas impacté les pratiques.

Dans notre cas, il nous faut par exemple acter que la catégorie professionnelle des formateurs d'enseignants de cycle 2 en France présente aujourd'hui une hétérogénéité assez importante, du point de vue de la fonction, de la connaissance de la didactique de l'écriture, de la connaissance du métier de professeur des écoles, du cycle visé ou du métier de formateur. En effet, cette catégorie regroupe des enseignants-chercheurs et des professeurs de lettres du second degré plus ou moins familiarisés avec ce niveau scolaire comme avec la didactique de l'écriture, des inspecteurs le plus souvent généralistes, des conseillers pédagogiques très expérimentés ou faisant nouvellement fonction sans la certification de formateur, des professeurs des écoles maitres formateurs (PEMF) ayant ou non enseigné dans ce cycle et approfondi les questions d'enseignement et d'apprentissage de l'écrit. Ignorer cette pluralité au profit d'une représentation homogénéisante et globalisante des utilisateurs ne peut qu'engendrer des obstacles dans l'appropriation et dans la diffusion des outils.

À l'inverse, au niveau de la conception des ressources, la prise en compte de l'hétérogénéité $\mathrm{du}$ réel incite à expliciter davantage, et dans un langage compréhensible pour des non-initiés, l'arrière-plan conceptuel, les soubassements méthodologiques, la démarche, les variantes, les passages à risque (Centre Alain Savary, 2017). Elle amène aussi à préciser ce qu'il faut faire et savoir en amont, en vue du déploiement de la formation. Cette valorisation du travail en amont rejoint l'importance de la planification du travail relevée par Dolz et Gagnon dans leur analyse des composantes majeures de la formation à l'enseignement de la production écrite (2018). Plutôt que de lister seulement des pré-requis, l'attention à l'hétérogénéité associée à une volonté de limiter la charge de travail pour l'utilisateur conduit également à inclure parmi les ressources différents apports synthétiques optionnels, importants pour l'action envisagée et susceptibles de faire monter en compétence les utilisateurs. Il s'agit par exemple de modélisations du processus scriptural valables pour ce niveau scolaire (notamment) et éclairant ou questionnant les manières d'enseigner (annexe 2), ou d'une synthèse des leviers majeurs pour rendre la complexité scripturale abordable au CP (annexe 3). Enfin, considérant, dans la lignée du CAS (2017) et d'Yvon et Saussez (2010) que le métier de formateurs demande d'ajuster son action selon le contexte (public, besoins de métier exprimés, imprévus, demandes institutionnelles, nombre de formés, durée de la formation ...) et de prendre en compte les passages à risque liés à l'objet (ou aux représentations de l'objet en présence), liés à l'approche adoptée ou aux dilemmes de métier, figurent aussi dans le tableau de 
travail ${ }^{7}$ que nous avons collectivement conçu pour élaborer les scénarios de formation des colonnes dédiées à ces éléments.

10 Au-delà de ce point précis, le dépliage des dimensions d'utilité, d'utilisabilité et d'acceptabilité en ergonomie, ou leur adaptation dans une perspective didactique (Goigoux, Renaud et Roux-Baron, 2019), montrent combien il est complexe de concevoir des ressources en cohérence avec le terrain. Dans notre cas, cette complexité est d'autant plus forte que le terrain est double, puisqu'il s'agit de la formation et de la classe.

11 En effet, on est bien face à une démarche exigeante quand sont pointés, en tant qu'éléments construisant et conditionnant l'utilisabilité (et parfois aussi l'acceptabilité), la nécessaire intégration des contraintes matérielles, organisationnelles, spatiales et temporelles des acteurs et des institutions ainsi que le besoin de flexibilité des outils pour un meilleur ajustement contextuel. Il en va de même quand l'acceptabilité passe par le respect du prescrit et par la prise en compte des habitudes de travail, des croyances et du système de valeurs des acteurs. Loin de tout applicationnisme, cette démarche consiste à placer les destinataires au cœur de la conception des outils. C'est pourquoi elle n'est possible qu'avec une bonne connaissance et compréhension des métiers de formateurs et d'enseignants (et plus globalement du terrain dans ses multiples dimensions), de même qu'avec un travail de conception en dialogue avec et au plus près de ce terrain (Béguin, 2013), dialogue sur lequel je reviendrai dans le point suivant.

12 Même si je les ai pointées distinctement pour la clarté du propos, la validité scientifique tout comme la validité et la pertinence de terrain participent conjointement de la qualité des ressources visées par le processus d'ingénierie didactique ici envisagé. En conséquence, il n'y a de robustesse qu'à l'aune de cette double exigence.

13 Néanmoins, dans les faits il n'est pas rare de voir, en situation de prolongement de résultats de recherche, des applications pour la formation ou la classe qui ne s'inscrivent pas ou qu'assez partiellement dans cette double exigence, souvent parce qu'elles généralisent ou appliquent à une grande échelle des résultats obtenus dans un contexte spécifique ou restreint. Dans le débat actuel sur les recherches menées en éducation, c'est une des limites que signale Bryk lorsqu'il pointe « la différence entre la connaissance que quelque chose peut fonctionner et la connaissance sur la façon de le faire fonctionner de manière fiable sur des contextes et des populations variés » (2017 : 14).

14 Il est également assez fréquent de trouver des manuels ou des outils à destination des enseignants et parfois des formateurs, qui tiennent compte de besoins et d'habitudes de travail du terrain, mais qui véhiculent ou mettent en œuvre des conceptions de l'écriture et de son apprentissage en contexte scolaire demeurant en tension avec les connaissances actuelles. À titre d'exemple, encore aujourd'hui, on trouve régulièrement l'écriture placée en aval ou en subordination de la lecture. Certains manuels donnent aux tâches d'écriture une place réduite ou un positionnement récurrent en fin de séance ou de séquence à la suite de tâches de lecture alors que ces deux composantes de l'écrit s'influencent et que l'écriture précoce peut avoir un effet bénéfique sur la conceptualisation de la langue écrite et plus globalement sur les compétences à l'écrit (Repères, 2013 ; Brissaud et al., 2016, par exemple).

15 Ces quelques constats soulignent combien, au niveau de la production de ressources, le véritable défi en matière de robustesse tient dans sa mise en œuvre. Car si les 
ressources élaborées ne sont robustes qu'à la condition d'être le fruit de cette double exigence scientifique et de terrain, leur robustesse ne peut s'obtenir via deux étapes distinctes ou en négligeant l'un des deux axes : elle repose sur l'emboitement et la mise en œuvre conjointe de ces deux exigences alors posées comme complémentaires et devant faire l'objet d'une coordination.

Dans cette perspective, le rôle du didacticien engagé dans un processus d'ingénierie didactique consiste, d'une part, à contribuer à la mise en œuvre de cette coordination permettant de conférer aux outils de la robustesse et, d'autre part, à l'étudier, en prêtant attention aux tensions, aux contraintes, aux conditions facilitatrices et aux bénéfices générés. Percevoir de la sorte le rôle du didacticien demande de ne pas limiter la didactique à une discipline scientifique productrice de connaissances sur l'enseignement et l'apprentissage de contenus disciplinaires ou de matières scolaires. En effet, il s'agit d'aussi inclure, dans la pertinence scientifique, la production de ressources pour l'action, dans une visée praxéologique d'ailleurs mise en exergue par nombre de didacticiens ou chercheurs engagés en didactique.

17 Cette optique amène également à ne désigner par "ingénierie didactique " ni l'ensemble des dispositifs ou moyens mis en œuvre dans une classe ou dans une formation, ni la seule construction et mise en œuvre de ces dispositifs ou de ces moyens. Positionnée comme une des missions du didacticien, il s'agit de considérer l'ingénierie didactique, de l'investir et de la valoriser en tant que type de recherche incluant l'étude des processus d'élaboration et de mise en œuvre des moyens d'enseignement et de formation (Artigue, 2002 ; Sénéchal, 2018). Une étude scientifique de ce type passe, entre autres, par l'analyse des exigences, des démarches et des conditions nécessaires pour obtenir des outils de qualité. Dans la tension constitutive en didactique entre exigence théorique et inscription dans la pratique, ce point de vue m'incite à souligner le déplacement opéré quand on considère la robustesse des ressources à l'aune de cette double exigence nécessairement conjointe.

\section{Caractéristiques des recherches de type collaboratif et robustesse}

Dans la perspective d'ingénierie didactique précisée ci-dessus, l'observation du cheminement collectif dans la production de ressources m'incite à analyser les formes que prennent la collaboration et le rôle de celle-ci pour construire des ressources robustes. Pour ce faire, je n'entrerai pas dans les débats sur la catégorisation des types de recherche et je ne chercherai pas à situer la recherche collaborative au sein de la constellation également formée par les recherches dites action, participative, partenariale, design, écologique, innovation, technologique, etc. Il m'a paru en effet plus fécond, pour répondre à mon objectif, de centrer l'observation sur les caractéristiques de ce type de recherche, recherche qui est à même de jouer un rôle majeur dans l'élaboration d'outils robustes. Pour la clarté du propos, je distinguerai quatre dimensions qui, dans les faits, comme le montrera l'analyse, interagissent grandement.

\section{Mixité, interactions et complémentarité polyphonique}

19 La recherche collaborative repose, par définition, sur une équipe et sur les interactions et la collaboration entre les différents acteurs composant cette équipe. De manière 
générale y prédomine une mixité (professionnelle, fonctionnelle, expérientielle, institutionnelle...) qui permet l'enrichissement mutuel par la confrontation et la complémentarité des points de vue, des questions, des regards interprétatifs, des compétences (Bednarz, 2013 ; Desgagné et al., 2001).

Dans certaines configurations, l'équipe compte uniquement des praticiens, enseignants ou formateurs (non chercheurs) par exemple, réunis autour de préoccupations professionnelles partagées et soucieux de s'inscrire ensemble dans une démarche de recherche (non scientifique). Cette logique où la recherche prend la forme d'une activité de compréhension et d'explication de la praxis par les groupes eux-mêmes, s'est répandue entre autres via les communautés d'apprentissage ou de pratique (Bryk, 2017).

21 Si de telles configurations rappellent que la dynamique de recherche n'est pas propre à la profession de chercheur (Vinatier et Morrissette, 2015) et que les deux gagnent à être distingués, dans notre cadre d'ingénierie didactique, la présence du chercheur n'est pas optionnelle, tout comme ne l'est pas celle du formateur et du formé. En effet, pour construire une rencontre entre les exigences de la recherche et celles de la formation et de la classe, la condition de base est que ces métiers soient représentés au sein de l'équipe de travail.

Dans la mesure où la diversité et la mixité sont posées et vécues comme moteur et source de complémentarité polyphonique permettant une meilleure appréhension de la complexité, il est intéressant que le métier de formateur, dont j'ai évoqué précédemment l'hétérogénéité, soit représenté de façon plurielle. Ainsi, au sein du groupe de concepteurs ${ }^{8}$, également expérimentateurs et tous formateurs, on note plusieurs catégories professionnelles, des rattachements institutionnels complémentaires et des ancrages de terrain différents (université, circonscriptions, zone d'éducation prioritaire, zone urbaine, périphérique ou rurale). Cette équipe présente aussi une complémentarité par les expériences, les parcours, les compétences en présence: spécialisation en didactique de l'écriture, formation en didactique du français, engagement dans des recherches-formations, expériences dans la formation de formateurs et dans la conception et le déploiement de formations auprès de publics variés.

Dans l'ingénierie menée, la mixité et la complémentarité ou construction polyphonique sont également assurées par des rencontres régulières entre notre groupe de concepteurs-expérimentateurs de ressources sur l'enseignement de l'écriture et les deux autres équipes de travail concevant des outils sur l'enseignement de la compréhension et de l'étude de la langue ainsi qu'avec des collègues du $\mathrm{CAS}^{9}$ spécialisés dans la formation et dans la production de ressources. Ces échanges entre collègues impliqués dans un travail du même ordre mais sur des objets différents ont largement contribué à nourrir le travail.

24 Enfin, la mixité a également pris corps par le travail mené avec des collègues formateurs et enseignants ${ }^{10}$ qui ont accepté de tester des ressources, d'être filmés ou interrogés dans leur classe et/ou leur formation pour nous permettre de préélaborer les ressources puis de progressivement les affiner. En termes de robustesse, la collaboration ponctuelle ou continue avec ces nombreux collègues aux profils variés (débutants ou expérimentés, courte ou longue expérience au $\mathrm{CP}$, réflexion déjà engagée ou non sur l'enseignement de l'écriture...) a aussi joué un rôle déterminant tout au long du processus. 


\section{Intelligence collaborative, démarche de pas-de-côté et distance critique} processus de recherche. comprendre ceux d'autrui. pertinence et validité sociales et scientifiques.

Revenons sur les interactions et l'échange des points de vue. Selon les recherches et les rôles des personnes qui y sont impliquées, la collaboration peut prendre des formes différentes, allant du "simple " échange d'informations en vue de l'enrichissement mutuel à une organisation et une avancée de la recherche basées sur les interactions et les points de vue polyphoniques. Si l'on se réfère à la mixité et à l'organisation de notre recherche présentée ci-avant, on peut en déduire que notre protocole prend appui sur des formes de collaboration différentes qui, combinées, font que cette ingénierie didactique repose sur la collaboration vue comme un évènement créatif dans le

Pour faire de l'intelligence collaborative le moteur de la recherche et pour que la complémentarité polyphonique aboutisse à une co-construction des ressources (ce que je détaillerai plus loin), il est nécessaire que les concepteurs-expérimentateurs adoptent une démarche de pas-de-côté par rapport à leur métier, leur expérience, leurs habitudes interprétatives, leur point de vue ou leur valeur, pour découvrir et

Ce déplacement passe par une prise de distance critique, elle-même renforcée par la confrontation ou le croisement des points de vue dont Bednarz (2013), entre autres, souligne le rôle prépondérant. Cette distance critique, souvent mentionnée dans les recherches de type collaboratif ou participatif (Verspieren, 2002; Anadon, 2013) est cohérente avec la visée qualitative de robustesse de l'ingénierie didactique, qui ne peut se faire sans compréhension des utilisateurs visés, sans connaissances sur l'activité de formation et d'enseignement, sur l'objet à enseigner et à apprendre, sur la didactique et sur les méthodes de recherche. Plus généralement, la prise de distance semble indispensable pour élaborer des outils nuancés et des réponses critiques coordonnant

Dans notre protocole, cette démarche s'est réalisée par la construction au sein du groupe d'une culture scientifique commune via l'échange de références et par des moments de travail avec des collègues plus expérimentés (notamment du CAS pour le volet production de ressources). Cette logique de pas-de-côté a aussi été mise en place par de nombreuses analyses croisées de corpus ainsi que par des interventions communes nous obligeant à croiser et à justifier en amont nos propositions.

De ce tissage des regards, des postures, des savoirs expérientiels ou savants ainsi que des valeurs qui forment la complémentarité polyphonique, résulte une extension des cultures, des compétences et des rôles, permettant à tous les concepteursexpérimentateurs de monter en compétences dans le travail distancié de production de ressources. Cette extension ne signifie pas que les compétences spécifiques des uns et des autres sont gommées au profit d'un partage total des rôles et des tâches, partage qui a minima réduirait ou compliquerait l'obtention de robustesse. Au contraire, l'invitation à changer de point de vue et à élargir sa palette de connaissances et de compétences prend appui sur les différences et la mixité au sein du groupe (restreint des concepteurs-expérimentateurs et élargi avec tous les autres contributeurs). 


\section{Interactions inscrites dans la durée et appui sur un laboratoire de terrain étendu et évolutif} interactions entre le pôle scientifique et celui de la formation et de l'enseignement. Comme relevé dans les travaux sur la recherche-action (Kervyn, 2011), ce type de collaboration ou d'interaction repose sur le principe selon lequel recherche et action s'enrichissent mutuellement, dans des trajectoires qui s'entrecroisent diversement selon les situations. Dans la recherche de robustesse, cette articulation est centrale pour éviter toute juxtaposition ou prise en compte insuffisante des contraintes scientifiques ou de terrain. Aussi vais-je expliciter sa mise en œuvre.

31 En préambule, il est important de signaler que ce ne sont pas les catégories professionnelles qui déterminent la représentation des deux pôles mais les connaissances et compétences effectives de chaque membre du groupe. Certes, l'enseignante-chercheuse que je suis a plus d'expertise dans la mise en œuvre d'une méthodologie de recherche rigoureuse ou dans l'écriture scientifique. Mais dans une logique de construction d'une culture partagée, il est par exemple commun que chacun fasse part de ses lectures ou de ses observations, signale des concepts ou travaux susceptibles de nourrir la production de ressources. Cette horizontalité dans la coconstruction, facilitée par l'expérience des pratiques collaboratives, n'empêche pas que chacun prenne part à la recherche avec un ou des rôles parfois spécifiques (relation avec le rectorat et la DSDEN, coordination du projet, prise en charge de la production d'un des scénarios de formation, expérimentation...). déjà notée, on constate de nombreux allers-retours entre le travail mené au sein du groupe restreint ou au sein des sous-groupes de concepteurs d'un des scénarios de formation et les prises de données et expérimentations dans les classes et en formation. Autrement dit, il ne s'agit pas de transposer ou d'opérationnaliser les résultats de la recherche par une collaboration en temps restreint dans quelques classes : on est ici dans un processus de production inscrit dans la durée car fait de tâtonnements exploratoires cadrés, d'ajustements successifs auprès de publics différents.

Il s'agit ainsi de constituer un véritable laboratoire de terrain varié et évolutif en fonction des besoins. À titre d'exemple, le travail mené avec des classes de CP dédoublés a ensuite été réalisé en classes « ordinaires ». Cette évolution du laboratoire va permettre d'observer les modifications, les tensions ou difficultés dans ces nouveaux contextes. Tel qu'envisagé, le laboratoire de terrain est aussi composé de lieux, de collègues, de classes, de groupes de formation avec lesquels les concepteurs vont pouvoir travailler, échanger, expérimenter. En cohérence avec la perspective collaborative, ce laboratoire inclut les concepteurs, concepteurs d'ailleurs eux-mêmes à maintes occasions expérimentateurs de leurs propres ressources ou de celles d'autres concepteurs de l'équipe.

allers-retours étendus, en termes de temps et de laboratoire de terrain, sont nécessaires si l'on souhaite élaborer des ressources robustes non pas pour quelques enseignants ou formateurs directement impliqués dans l'ingénierie didactique (Artigue, 2002) mais pour un public plus large que vise la mise à disposition gratuite sur un site internet comme celui du $\mathrm{CAS}^{11}$. Par rapport aux recherches collaboratives dont l'objectif est d'outiller les acteurs qui participent à la recherche, le principe de 
conception continue dans l'usage (Goigoux, 2017) au travers d'utilisateurs différents, dans lequel nous nous inscrivons modestement, tend à répondre au besoin de recomposition pour un ajustement optimal aux utilisateurs visés.

\section{Une collaboration sous forme de co-élaboration initiale et continue}

Pour approfondir le point précédent et analyser la forme que prend la collaboration dans notre perspective d'ingénierie didactique, je voudrais préciser la portée de la coélaboration visant à doter les ressources de robustesse. Comment prend forme la coconstruction impliquée dans la mise en œuvre conjointe des deux exigences (scientifique et de terrain) au cœur de la robustesse ? Sur quoi porte-t-elle et comment se manifeste-t-elle?

Quand on retrace l'historique du projet d'ingénierie didactique mené, la collaboration apparait au cœur du démarrage de la recherche. En effet, lors de la première rencontre, le travail avec l'équipe alors en constitution a porté sur les résonances des résultats de la recherche LireÉcrireCP avec les besoins du terrain : besoins de chacun en tant que formateur, besoins des collègues et des formés avec qui chacun exerce, besoins des élèves. En repartant des besoins des terrains représentés et en les mettant en parallèle avec les résultats de la recherche, nous avons listé ce qu'il nous paraissait intéressant d'investiguer. Chacun s'est ensuite positionné sur ce qui faisait le plus sens pour lui en fonction de son environnement professionnel, de ses contraintes, des collaborations existantes... C'est ainsi que nous nous sommes lancés dans la production de différentes ressources pertinentes pour les uns et les autres et au vu des résultats de la recherche. Rendre partagés ces choix initiaux ainsi que l'orientation du travail a un fort impact sur l'engagement et l'implication, et revient à fonder ce que Verspieren appelle l'acteur collectif (2002), mettant ici encore en œuvre l'horizontalité du travail basé sur le principe d'égalité.

Par la suite, certains choix ont évolué, notamment au vu des besoins et retours émanant du laboratoire de terrain. À titre d'exemple, le scénario sur l'enseignement de l'encodage de phrases devait au départ porter sur l'évaluation de la production écrite. $\mathrm{Au}$ fil des observations, nous nous sommes rendu compte que la question de l'évaluation seule n'avait que peu de pertinence si les enseignants ne mettaient pas en place régulièrement des situations de production écrite (Repères, 2015). Le mode de travail conjoint qui a permis ces évolutions s'est globalement renforcé dans la mesure où il s'est étendu, au fil des occasions et des besoins, à d'autres dimensions comme le partage des tâches et l'organisation du travail, collectivement validés, la structuration et la forme des ressources, longuement négociées, la formation et la composition du laboratoire de terrain. Cette co-construction, qui va jusqu'à la co-validation des ressources et différentes situations orales et écrites de co-énonciation, confère aux outils et aux discours un caractère métissé (Miguel Addisu et Maire-Sandoz, 2018) participant grandement à leur robustesse. En accord avec Bourassa et Boudjaoui (2012) ou Sénéchal (2018), on peut penser que ces deux dernières dimensions sont révélatrices de l'importance de ce mode de fonctionnement conjoint. Le travail partagé durant ces années a amené une extension d'expertise qui permet aux uns et aux autres de présenter à leur manière le travail et de co-valider les ressources, en s'appuyant sur des compétences amplifiées, compétences finalisant le principe de l'intelligence collaborative. 
L'intelligence collaborative et la co-élaboration telles que décrites sont, je tiens à le souligner, régies par une valeur d'égalité. Celle-ci se réalise par la non-supériorité de la recherche sur le terrain (et vice versa) - non supériorité aussi mise en avant dans la robustesse - et par le respect des compétences sans hiérarchisation de celles-ci. Privilégier l'horizontalité dans le travail et les relations plutôt qu'une organisation descendante liée aux statuts académiques permet également de donner forme à cette valeur d'égalité. Par ailleurs, si l'horizontalité nourrit l'engagement professionnel (Anadon, 2013), l'implication, plusieurs fois signalée, est indispensable pour mener une recherche collaborative de longue durée, s'ajoutant au travail quotidien, apportant peu de reconnaissance universitaire mais un gain réel à la fois collectif et personnel.

\section{Pour conclure}

Face à la pluralité nécessaire des recherches existant aujourd'hui en didactique des langues et des cultures, et plus spécifiquement ici en didactique du français et de l'écriture, quand et à quelles conditions opter pour une recherche de type collaboratif ? Pour apporter un élément de réponse à cette question vive en didactique, a été étudié un dispositif d'ingénierie en mettant en exergue des dimensions des recherches dites collaboratives, susceptibles d'assurer un rôle clé dans l'obtention de ressources robustes.

Par un dialogue entre observation du processus de recherche engagé et travaux nécessairement pluriels, l'analyse menée permet de montrer comment les caractéristiques et les principes de la recherche collaborative mis en avant constituent une réponse cohérente à une question cruciale en didactique, à savoir comment rendre des ressources robustes. Complexe à construire, cette exigence de robustesse nécessite l'appui sur des connaissances et des savoirs d'expérience mixtes, un tissage des points de vue et une complémentarité polyphonique, un laboratoire de terrain étendu, une coélaboration initiale et continue, des tâtonnements exploratoires cadrés et des ajustements successifs inscrits dans la durée, une implication et une distanciation critique ainsi qu'une horizontalité maximale. Autant de dimensions que peut fournir l'inscription dans une recherche de type collaboratif qui elle aussi refuse «le divorce classique entre théorie et pratique » (Vinatier, $2014: 250$ ).

41 Sans prétendre qu'il s'agisse là de la seule voie appropriée, le pari de l'intelligence collaborative déclinée en processus de recherche balisé et exigeant apparait au final comme un gain indéniable pour la formation comme pour les recherches en didactique.

\section{BIBLIOGRAPHIE}

Anadon, M. (2013). Recherche participative. In I. Casillo, avec R. Barbier, L. Blondiaux, F. Chateauraynaud, J-M. Fourniau, R. Lefebvre, C. Neveu \& D. Salles (dir.), Dictionnaire critique et 
interdisciplinaire de la participation. Paris, GIS Démocratie et Participation. En ligne : http:// www.dicopart.fr/fr/dico/recherche-participative

Artigue, M. (2002). Ingénierie didactique : quel rôle dans la recherche didactique aujourd'hui ? Les dossiers des sciences de l'éducation, $\mathrm{n}^{\circ}$ 8, p. 59-72.

Bednarz, N. (2013). Recherche collaborative et pratique enseignante : Regarder ensemble autrement. Paris, l'Harmattan.

Béguin, P. (2013). La conception des instruments comme processus dialogique d'apprentissages mutuels. In P. Falzon (éd.), Ergonomie constructive, Paris, PUF, p. 147-160.

Bourassa, B \& Boudjaoui, M. (dir.) (2012). Des recherches collaboratives en sciences humaines et sociales : enjeux, modalités et limites. Québec, Presses de l'Université Laval.

Brissaud, C., Pasa, L., Ragano, S. \& Totereau, C. (2016). Effets des pratiques d'enseignement de l'écriture au cours préparatoire. Revue française de pédagogie, 196, p. 85-100.

Bryk, A. S. (2017). Anthony S. Bryk, Accélérer la manière dont nous apprenons à améliorer. Éducation et didactique, vol. 11-2, p. 11-29.

Centre Alain Savary (2017). Concevoir des formations pour aider les enseignants à faire réussir tous les élèves. Lyon, ENS de Lyon (version 6, novembre 2017).

Desgagné, S., Bednarz, N., Couture, C., Poirier, L. \& Lebuis, P. (2001). L'approche collaborative de recherche en éducation : un rapport nouveau à établir entre recherche et formation. Revue des sciences de l'éducation, 27-1, p. 33-64.

Dolz, J. \& Gagnon, R. (dir.) (2018). Former à enseigner la production écrite. Lille, Presses universitaire du Septentrion.

Goigoux, R. (dir.) (2016). Étude de l'influence des pratiques d'enseignement de la lecture et de l'écriture sur la qualité des apprentissages au cours préparatoire. Rapport de recherche. Lyon, Institut français d'Éducation. En ligne : http://ife.ens-lyon.fr/ife/recherche/lire-ecrire/rapport.

Goigoux, R. (2017). Associer chercheurs et praticiens à la conception d'outils didactiques ou de dispositifs innovants pour améliorer l'enseignement. Éducation \& didactique, vol. 11-3, p. 135-142.

Goigoux, R., Renaud, J. \& Roux-Baron, I. (2019). Conception continuée dan l'usage d'outils didactiques innovants au service de l'amélioration des apprentissages des élèves : un cadre théorique et deux études empiriques. Communication au REF, Montpellier, juillet 2019.

Kervyn, B. (2008). Didactique de l'écriture et phénomènes de stéréotypie. Le stéréotype comme outil d'enseignement et d'apprentissage de l'écriture en fin d'école primaire. Thèse de doctorat, Louvain-laNeuve, Université de Louvain, vol. 1.

Kervyn, B. (2011). Caractéristiques et pertinence de la recherche-action en didactique du français. In B. Daunay \& B. Schneuwly (dir.) Les concepts et les méthodes en didactique du français. Namur, Presses Universitaires de Namur, p. 194-224.

Miguel-Addisu, V. \& Maire-Sandoz, M.-O. (2018). Enseigner la lecture dans une classe plurilingue et défavorisée. In S. Briquet (dir.), Enseignement-apprentissage de la lecture, Carrefour de l'Éducation, $\mathrm{n}^{\circ} 46$, p. $15-26$.

Repères (2013). Premières pratiques d'écriture : état des recherches francophones. Lyon, ENS Éditions, $n^{\circ} 47$.

Repères (2015). Lecture et écriture : le choix des enseignants au début de l'école élémentaire. Lyon, ENS Éditions, $n^{\circ} 52$. 
Revue française de Pédagogie (2016). Apprendre à lire et à écrire au cours préparatoire. Lyon, ENS Éditions, $n^{\circ} 196$.

Sénéchal, K. (2018). Impacts d'une collaboration avec des enseignants sur l'élaboration et la validation du produit d'une ingénierie didactique. Recherches qualitatives, vol. 37, nº 2, p. 128-149.

Tricot, A., Plégat-Soutjis, F., Camps, J.-F., Amiel, A., Lutz G. \& Morcillo, A. (2003). "Utilité, utilisabilité, acceptabilité : interpréter les relations entre trois dimensions de l'évaluation des EIAH". In C. Desmoulins, P. Marquet \& D. Bouhineau, Environnements Informatiques pour l'Apprentissage Humain, Strasbourg, France. ATIEF, INRP, p. 391-402.

Verspieren, M.-R. (2002). Quand l'implication se conjugue avec distanciation : le cas de la recherche-action de type stratégique. Étude de communication, $n^{\circ} 25$, p. 105-123.

Vinatier, I. (2014). Recherches collaboratives. In A. Jorro, Dictionnaire des concepts de la professionnalisation, Louvain-la-Neuve, De Boeck, p. 249-252.

Vinatier, I. \& Morrissette, J. (2015). Les recherches collaboratives : enjeux et perspectives. Carrefours de l'éducation, n 39-1, p. 137-170.

Yvon, F \& Saussez, F. (2010) (dir.). Analyser l'activité enseignante. Des outils méthodologiques pour l'intervention et la formation, Lyon, PUL.

Annexe $1:$ Architecture globale du travail de production de ressources sur la formation à l'enseignement de l'écriture

\section{ANNEXES}

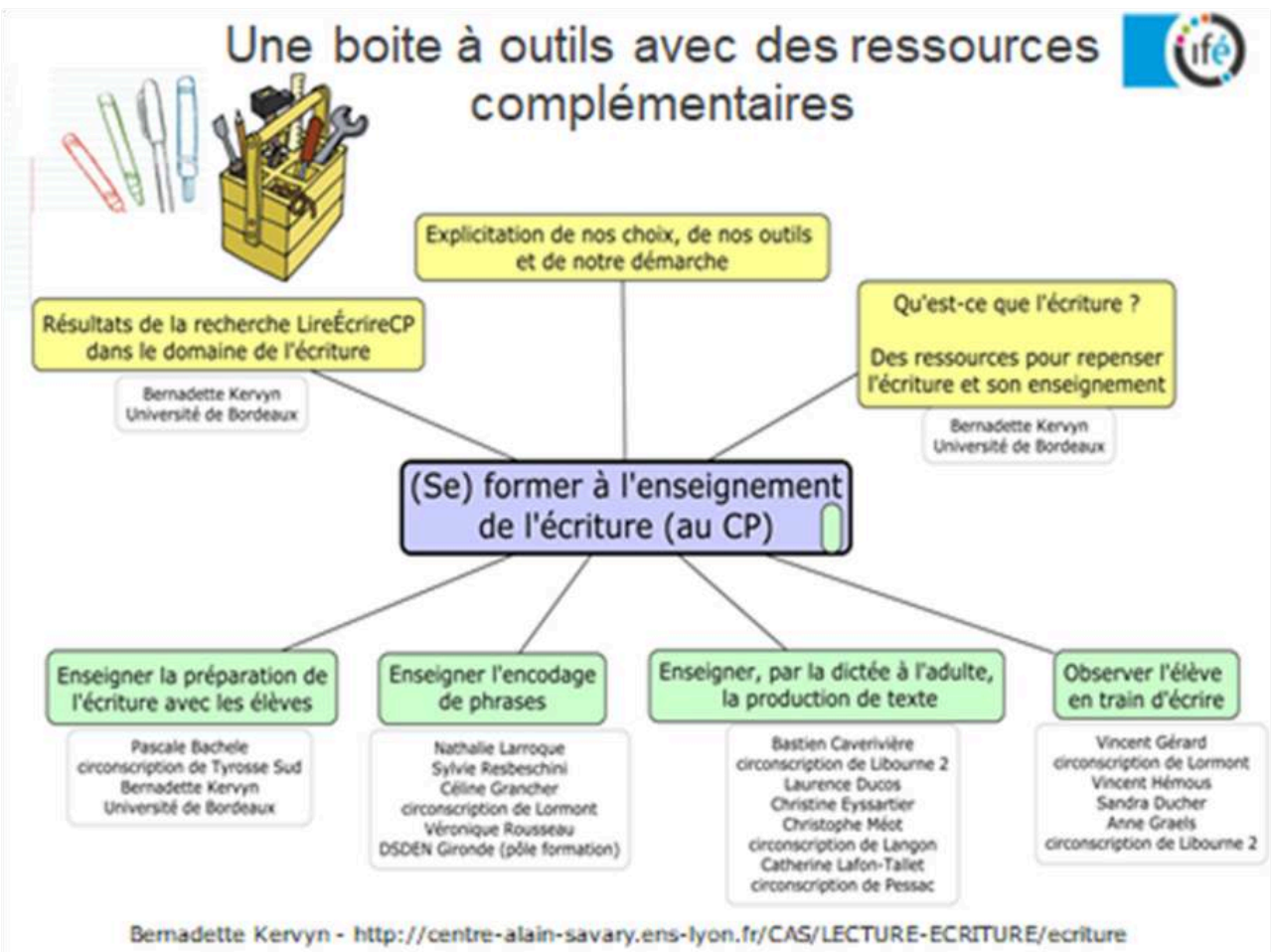

Annexe 2 : Modélisation de l'activité scripturale 


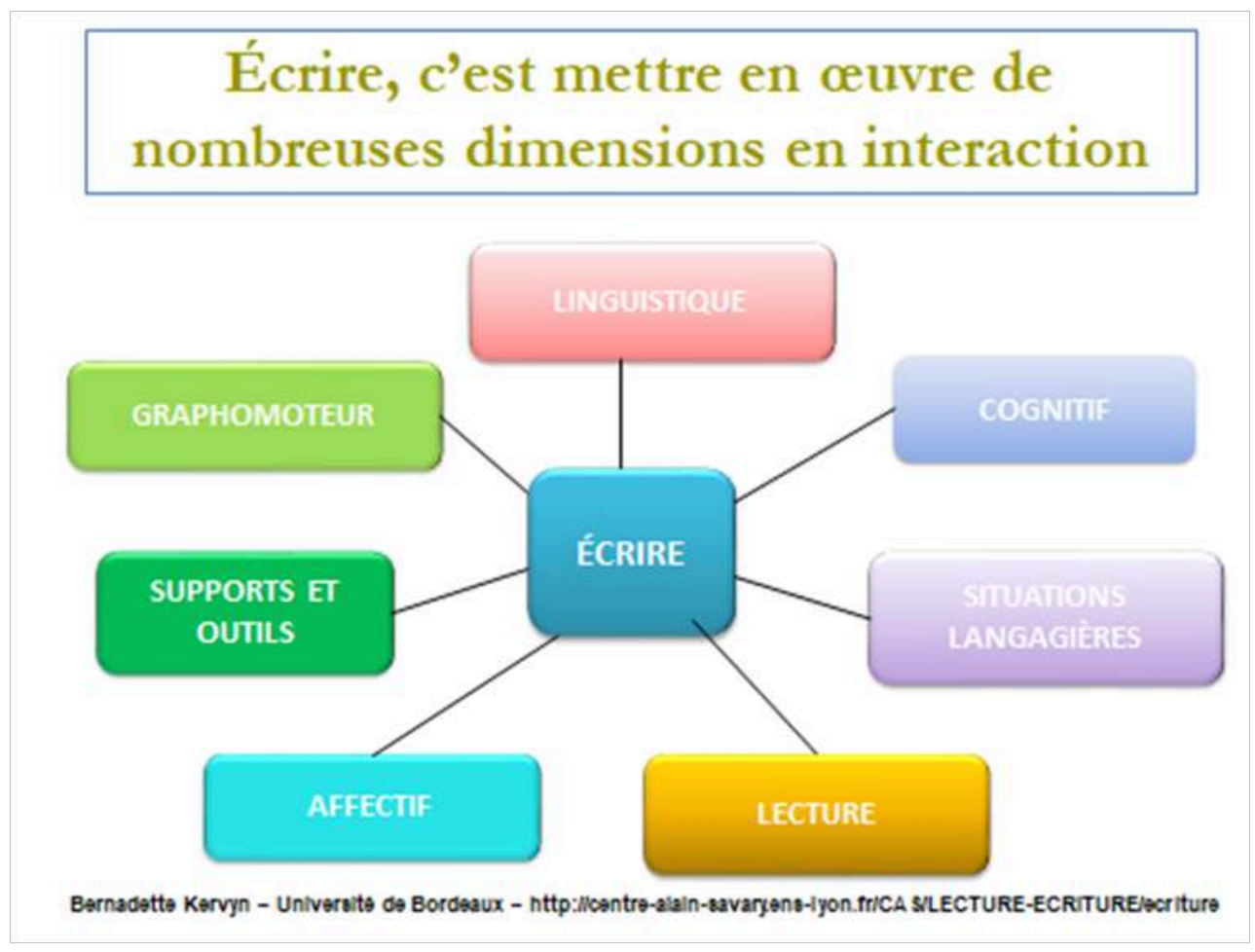

\section{NOTES}

1. Malgré l'usage fréquent du pronom «nous » en recherche pour se désigner en tant qu'auteur et parfois induire une intention de neutralité axiologique, certes toute relative, dans le présent article, choix est fait de recourir à une écriture en «je " quand il s'agit de l'auteur. Ce choix permettra de réserver l'usage du "nous » pour désigner le collectif engagé dans la recherche dont il sera question. Seront aussi utilisés de façon distincte le «nous » et le « on », ce dernier visant à inclure les lecteurs potentiels.

2. Recherche menée de 2012 à 2016 et intitulée «Étude de l'influence des pratiques d'enseignement de la lecture et de l'écriture sur la qualité des premiers apprentissages au cours préparatoire ".

3. Pour une étude croisée des concepts de transposition et d'opérationnalisation.

4. Le travail des deux autres groupes porte sur la compréhension en lecture (coordonné par M.-F. Bishop) et sur l'étude de la langue (coordonné par P. Gourdet). Au quotidien, les 3 groupes ont travaillé de façon distincte mais avec environ 2 rencontres annuelles pour mutualiser et croiser le travail effectué au sein de chaque équipe.

5. L'architecture de ce travail est donnée dans l'annexe 1. Nous y reprenons le symbole de la boite à outils utilisé par M.-F. Bishop pour représenter le travail mené sur la compréhension.

6. http://centre-alain-savary.ens-lyon.fr/CAS/education-prioritaire/ressources/theme-1perspectives-pedagogiques-et-educatives/lire-ecrire-parler-pour-apprendre-dans-toutes-lesdisciplines/dossier-lire-ecrire/copy_of_ecriture-se-construire-une-culture-commune.

7. Ce document de travail est donné en annexe 4.

8. Il s'agit de Pascale Bachelé, Bastien Caverivière, Laurence Ducos, Christine Eyssartier, Vincent Gérard, Bernadette Kervyn, Nathalie Larroque, Sylvie Rebeschini et Véronique Rousseau, auxquels s'ajoutent Sandra Ducher, Anne Graëls, Sébastien Hémous et Christophe Méot pour la première moitié du projet et Céline Grancher ainsi que Catherine Lafont-Tallet pour la seconde moitié. Sincère merci à tous ces collègues. 
9. Dans l'ordre alphabétique Virginie Hohl, Frédérique Jarre, Frédérique Mauguen et Patrick Picard.

10. Sans tous les nommer, je tiens à remercier particulièrement Isabelle Bernard, Déborah Curuchet, Matthieu Deymard, Lucie Drouilhet, Alban Dubois, Peyo Goïgoetchea, Céline Lasserre, Béatrice Maheas, Anne-Laure Mazet, Mylène Mortier, Magali Piazza, Martine Pujolle, Aline Rispail-Hourcal.

11. Sur l'année 2018, les premières ressources déposées ont été visionnées environ 12000 fois.

\section{RÉSUMÉS}

Quelles sont les caractéristiques essentielles des recherches de type collaboratif qui contribuent à produire des ressources de formation de qualité ? Pour répondre à cette question en la situant dans le champ de la didactique du français, cet article prend comme corpus le processus d'ingénierie didactique engagé suite à la recherche LireÉcrireCP pour produire des outils sur l'enseignement de l'écriture au début de l'école élémentaire. À partir de ce corpus, est mis en avant le fait que la qualité de telles ressources repose sur une exigence conjointe de recherche et de terrain, qui leur confère de la robustesse. Dans un dialogue avec cette analyse du processus d'ingénierie didactique et en situant le propos au cœur des préoccupations praxéologiques de la didactique en tant que discipline productrice de connaissances et de ressources pour l'action, je mets en exergue différentes dimensions des recherches dites collaboratives en explicitant en quoi elles permettent de construire la robustesse visée.

What are the essential characteristics of collaborative research that contribute to the production of quality training resources? To answer this question by situating it in the field of French language didactics, this article takes as a corpus the process of didactic engineering initiated following the LireÉcrireCP research to produce tools on the teaching of writing at the beginning of elementary school. From this corpus, we highlight the fact that the quality of such resources is based on a joint requirement of research and field work, which gives them robustness. In a dialogue with this analysis of the didactic engineering process and by situating our subject at the heart of the praxeological concerns of didactics as a discipline producing knowledge and resources for action, we highlight different dimensions of so-called collaborative research by explaining how they enable the construction of the targeted robustness.

\section{INDEX}

Mots-clés : recherche collaborative, ingénierie didactique, didactique de l'écriture, robustesse, méthodes de recherche

Keywords : collaborative research - didactic engineering - didactics of writing - robustness research methods 


\section{AUTEUR}

\section{BERNADETTE KERVYN}

Université de Bordeaux - INSPE Académie de Bordeaux ; Lab-E3D - EA 744, MCF $7^{\mathrm{e}}$ section

Situés en en didactique, mes travaux portent sur l'acquisition du processus scriptural, sur la littéracie scolaire, sur le rôle des interactions langagières pour enseigner et apprendre, et sur les élèves en difficulté dans l'apprentissage du FLS-FLSCO. Mes recherches de type collaboratif m'amènent à interroger les démarches méthodologiques à même de produire des connaissances et des ressources en dialogue avec le terrain.

bernadette.kervyn[at]u-bordeaux.fr 